\title{
Proteinase Isoenzyme Patterns of Bacteroides nodosus: Distinction between Ovine Virulent Isolates, Ovine Benign Isolates and Bovine Isolates
}

\author{
By DALE EVERY \\ Wallaceville Animal Research Centre, Research Division, Ministry of Agriculture \& \\ Fisheries, Private Bag, Upper Hutt, New Zealand
}

(Received 10 June 1981)

\begin{abstract}
Bacteroides nodosus isolates from ovine virulent footrot and ovine benign footrot and bovine isolates of low virulence for sheep were distinguishable from each other by their proteinase isoenzyme patterns after polyacrylamide gel electrophoresis. Variants of low virulence were not always distinguishable from their virulent parent strains. The molecular weights of the isoenzymes ranged from 70000 to 129000 . The relationship of isoenzyme patterns to virulence is discussed.
\end{abstract}

\section{INTRODUCTION}

Bacteroides nodosus has been isolated from cases of ovine virulent footrot (Beveridge, 1941), ovine benign footrot (Thomas, 1962; Egerton \& Parsonson, 1969) and interdigital dermatitis in cattle (Laing \& Egerton, 1978). The latter two isolates do not cause virulent footrot in artificially challenged sheep, and therefore efforts to eradicate and prevent spread of these strains have been given low priority in virulent footrot control programmes in Australia (Thomas, 1962; Egerton \& Parsonson, 1969; Stewart, 1979). To assist these programmes, diagnostic tests based on proteinase characteristics have been developed to differentiate $B$. nodosus from cases of virulent and benign footrot (Thomas, 1962; Egerton \& Parsonson, 1969; Depiazzi \& Richards, 1979; Stewart, 1979). These tests do not distinguish between ovine benign isolates and bovine isolates, and it is not known whether cattle and sheep have their own specific strains of $B$. nodosus. It is also not known whether differences in proteinase characteristics observed in the diagnostic tests result from quantitative or qualitative enzyme differences.

This paper describes a method for producing proteinase zymograms which differentiate the two types of ovine isolates and the cattle isolates from each other, and shows that the various isolates have both quantitative and qualitative differences in their proteinase enzymes. An investigation is also made of a suggestion that benign $B$. nodosus isolates arise from virulent isolates.

\section{METHODS}

Bacterial strains and growth conditions. Bacteroides nodosus strains 65, 80, 91, 135, 137 and 144 were isolated from clinical cases of ovine footrot in New Zealand. Strains 141 and 142 were isolated from interdigital dermatitis in New Zealand cattle. Strain ATCC 25549, an ovine virulent isolate, was obtained from the American Type Culture Collection. Strains A198 from virulent footrot and A134, A178 and A305 from benign footrot were isolated from Australian sheep and were obtained from the McMaster Laboratory, Division of Animal Health, CSIRO, Sydney, Australia. The prefix A is used to distinguish them from New Zealand strains. Bacteroides nodosus variants $91 \mathrm{M}, 91 \mathrm{C}$ and A198M were derived from serially passaged liquid cultures of wild-type strains 91 
and A198 as described by Skerman et al. (1981). The virulence characteristics of these strains and culture variants in artificial challenge experiments have been described previously (Skerman et al., 1981).

The organisms were grown anaerobically at $37^{\circ} \mathrm{C}$ in liquid Trypticase/arginine/serine medium (Skerman, $1975)$ to maximum cell density $\left(1-3 \times 10^{8}\right.$ cells ml $\left.^{-1}\right)$ at the end of the exponential phase of growth.

Preparation of proteinase zymogram. Bacteria were removed from cultures by centrifugation and the supernatants were concentrated with Carbowax to give sufficient proteinase in $10 \mu \mathrm{l}$ of each sample to produce a clear reaction in the zymogram. The number of bacteria from which each extracellular proteinase sample was derived is shown in Fig. 1. Samples $(10 \mu \mathrm{l})$ were applied to a horizontal slab polyacrylamide gel for electrophoresis as described by Every $(1981 \mathrm{a})$. Electrophoresis in $7 \%(\mathrm{w} / \mathrm{v})$ acrylamide gel was for $5 \mathrm{~h}$ at $1000 \mathrm{~V}$ and $15-35 \mathrm{~mA}$, and proteinase isoenzymes were detected by a gelatin-in-agar overlay method (Every, 1981b). The overlay consisted of a $0.7 \mathrm{~mm}$ thick agar film $\left(1.5 \%\right.$, w/v) containing $0.5 \%$ (w/v) gelatin (Difco), $3 \mathrm{mM}^{-\mathrm{NaN}_{3}}$, $1 \mathrm{mM}-\mathrm{NiCl}_{2}$ and $20 \mathrm{~mm}$-Tris/ $\mathrm{HNO}_{3}$ buffer, $\mathrm{pH} \mathrm{8.8.} \mathrm{The} \mathrm{overlay} \mathrm{was} \mathrm{incubated} \mathrm{in} \mathrm{contact} \mathrm{with} \mathrm{the} \mathrm{polyacryl-}$ amide gel slab for $25 \mathrm{~min}$ at $37^{\circ} \mathrm{C}$. Zones of gelatin clearing in the agar film were made visible by precipitation of the unhydrolysed gelatin with saturated ammonium sulphate solution. The zymogram was photographed against a black background which gave the isoenzyme bands a dark appearance.

Molecular weight determination. Molecular weights of proteinase isoenzymes were calculated by the method of Slater (1969) using 9-17\% (w/v) gradient polyacrylamide gels, and the standard proteins from Pharmacia electrophoresis calibration kits, as described by Every $(1981 a)$. Electrophoresis of samples and standard proteins was for $16 \mathrm{~h}$ at $600 \mathrm{~V}$ and 7-25 mA. The isoenzymes were located directly in the polyacrylamide gel electrophoretogram by the following procedure. Whatman $3 \mathrm{MM}$ paper saturated with $50 \mathrm{mM}$-Tris $/ \mathrm{HNO}_{3}$ buffer ( $\mathrm{pH} 8.8$ ) containing Hammarsten casein $\left(\mathrm{BDH} ; 10 \mathrm{mg} \mathrm{ml}^{-1}\right.$ ) was placed on the area in the gel containing separated proteinase isoenzymes. After incubation for $20 \mathrm{~min}$ at $37^{\circ} \mathrm{C}$ the gel was stained with Coomassie Blue R250 (Wyckoff et al., 1977) to give a negative-staining effect: clear areas corresponding to isoenzyme bands appeared against a blue background of unhydrolysed stained casein.

\section{RESULTS AND DISCUSSION}

The results (Fig. 1 and Table 1) showed that all the B. nodosus strains and variants secreted a mixture of proteinase isoenzymes of various molecular sizes and that the ovine virulent isolates, ovine benign isolates and bovine isolates could be distinguished from each

Table 1. Molecular weights of B. nodosus proteinase isoenzymes

\begin{tabular}{|c|c|c|c|c|}
\hline \multicolumn{4}{|c|}{ Isoenzyme number } & \multirow{2}{*}{$\begin{array}{c}10^{-3} \times \\
\text { Molecular } \\
\text { weight }\end{array}$} \\
\hline $\begin{array}{l}\text { Ovine virulent } \\
\text { isolates }\end{array}$ & $\begin{array}{l}\text { Ovine benign } \\
\text { isolates }\end{array}$ & $\begin{array}{c}\text { Bovine isolate } \\
141\end{array}$ & $\begin{array}{c}\text { Bovine isolate } \\
142\end{array}$ & \\
\hline & & & $B^{\prime} 1$ & 70 \\
\hline Ov1 & Ob1 & B1 & . & 75 \\
\hline & . & B2 & . & 77 \\
\hline Ov2 & . & & . & 78 \\
\hline Ov3 & & B3 & . & 82 \\
\hline & $\mathrm{Ob} 2$ & & & 83 \\
\hline Ov4 & Ob3 & B4 & $\mathrm{B}^{\prime} 2$ & 88 \\
\hline . & Ob4 & B5 & & ND \\
\hline & Ob5 & & $B^{\prime} 3$ & 92 \\
\hline Ov5 & . & B6 & . & 94 \\
\hline Ov6 & . & & . & 97 \\
\hline . & 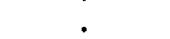 & B7 & . & 98 \\
\hline . & Ob6 & . & $B^{\prime} 4$ & 101 \\
\hline & Ob7 & . & . & ND \\
\hline Ov7 & . & 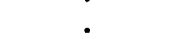 & . & 107 \\
\hline . & & B8 & . & 110 \\
\hline & Ob8 & . & $B^{\prime} 5$ & 114 \\
\hline Ov8 & & . & & 123 \\
\hline . & Ob9 & . & $B^{\prime} 6$ & 129 \\
\hline
\end{tabular}

ND, Not determined. Although isoenzymes Ob7 and B5 cannot be seen in Fig. 1, they were seen in trace amounts in the original zymograms and are known to be present at higher concentrations in the supernatants of early-exponential phase cultures (unpublished results). 


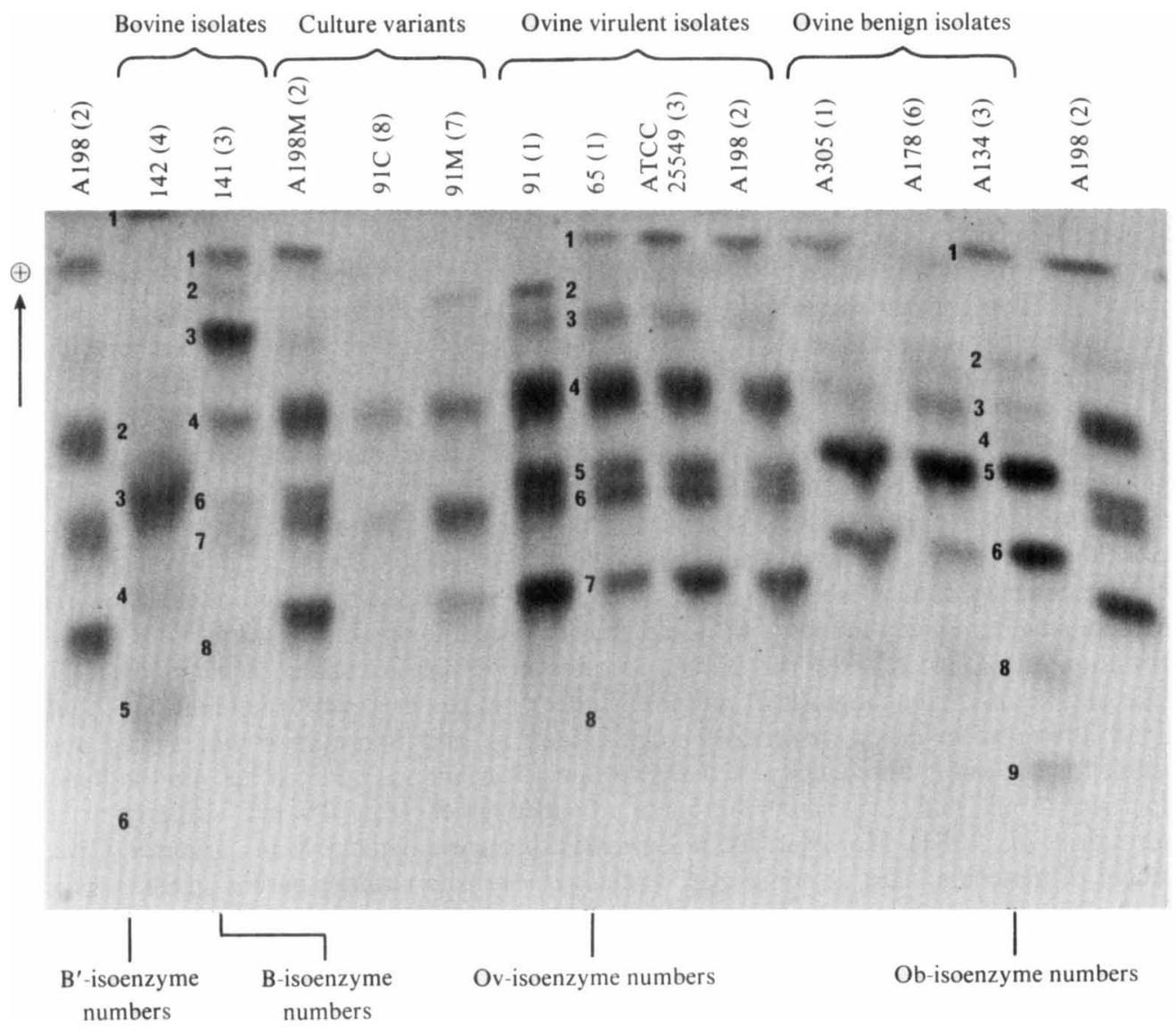

Fig. 1. Proteinase isoenzyme patterns after electrophoresis of culture supernatants of various B. nodosus clinical isolates and culture variants in $7 \%$ polyacrylamide gel. The numbers of the strains and variants are shown at the top of the zymogram with, in parentheses, the number of bacteria $\left(\times 10^{-7}\right)$ from which each extracellular proteinase sample was derived. The ovine virulent strain A198 has been included at both sides of the zymogram to make it easier to compare isoenzyme patterns. By extracting individual isoenzymes from the $7 \%$ polyacrylamide gel and re-running them on gradient gels, it was shown that the relative positions of isoenzymes in the two gel systems were the same. Thus, the isoenzyme number system used in this figure is the same as that in Table 1.

other by their proteinase isoenzyme patterns. The isoenzymes of all the virulent isolates had the same characteristic molecular weight distribution, but each isolate had different quantities of individual isoenzymes; this was especially noticeable with strain 91 . Other virulent isolates from New Zealand sheep (strains 80, 135, 137 and 144), not shown in Fig. 1, had similar isoenzyme patterns. Only two isoenzymes (Ov1 and Ov4) of the virulent isolates had the same molecular weights as isoenzymes (Ob1 and $\mathrm{Ob} 3)$ of the ovine benign isolates (Table 1). All the ovine benign isolates had similar isoenzyme molecular weight distributions, but they differed in the quantity of each isoenzyme. In contrast, the isoenzymes of the two bovine isolates 141 and 142 differed in molecular weight and in relative concentration (Table 1). Bovine isolate 142 had five isoenzymes ( $B^{\prime} 2, B^{\prime} 3, B^{\prime} 4, B^{\prime} 5$ and $\left.B^{\prime} 6\right)$ in common with ovine benign isolates, one isoenzyme $\left(\mathrm{B}^{\prime} 2\right)$ in common with virulent isolates, one novel isoenzyme $\left(B^{\prime} 1\right)$ and only one isoenzyme $\left(B^{\prime} 2\right)$ in common with bovine isolate 141 . Bovine isolate 141 had four isoenzymes (B1, B3, B4 and B6) in common with virulent isolates, three isoenzymes (B 1, B4 and B5) in common with ovine benign isolates, and possibly three novel isoenzymes (B2, B7 and B8). 
Since Beveridge (1941) first suggested that $B$. nodosus proteinase may be associated with pathogenesis of footrot in sheep, there have been several reports of a correlation between the virulence of $B$. nodosus and proteolytic activity on casein (Thomas, 1962; Egerton \& Parsonson, 1969), hide powder-azure (Depiazzi \& Richards, 1979) and elastin (Stewart, 1979; Skerman et al., 1981). The presence of low levels or the absence of certain Ov-type isoenzymes (Table 1) in the ovine benign isolates and bovine isolates may account for their low elastase activity (Stewart, 1979; Skerman et al., 1981) and the rapid loss of hide powder-azure degrading activity in stationary phase cultures (Depiazzi \& Richards, 1979; Stewart, 1979) in comparison to virulent isolates. This suggestion is currently being examined by comparative substrate specificity and enzyme stability studies using purified individual isoenzymes.

Variants $91 \mathrm{M}$ and $\mathrm{A} 198 \mathrm{M}$ have been shown to have low virulence characteristics similar to the ovine benign isolates (Skerman et al., 1981) and it was considered that the benign isolates might have arisen from virulent isolates in an analogous way to the production of culture variants from their parent virulent strains. In this case the $\mathrm{M}$-variants might be expected to have similar proteinase isoenzyme patterns to the benign isolates. In fact, the $\mathbf{M}$-variants had electrophoretically similar isoenzymes to their parent virulent strains, and although variant $91 \mathrm{M}$ had greatly reduced amounts of isoenzymes, the qualitative and quantitative isoenzyme distribution of variant A198M was almost identical to that of its parent virulent strain (Fig. 1). Hence, the in vitro changes in $B$. nodosus appear not to be relevant to conditions in vivo, and an alteration in other virulence factor(s), such as pili (Skerman et al., 1981), might account for the low pathogenicity of these variants. Variant $91 \mathrm{C}$, which had no pathogenic properties at all, had very small amounts of isoenzymes (Fig. 1) and virtually no pili (Skerman et al., 1981). However, the isoenzymes that were detectable in variant 91C were identical in molecular size to some of the isoenzymes in its virulent parent strain. It is not known whether the production of culture variants has a genetic basis, but the demonstration that the various clinical isolates produce different molecular forms of isoenzymes from each other shows that they are genetically distinct.

I thank T. M. Skerman for providing cultures of $B$. nodosus and R. S. Green for skilled technical assistance.

\section{REFERENCES}

Beveridge, W. I. B. (1941). Foot rot in sheep: a transmissible disease due to infection with Fusiformis nodosus (n.sp.). Studies on its cause, epidemiology and control. Bulletin of the Council of Scientific and Industrial Research of Australia, no. $140,1-56$.

Depiazzi, L. J. \& RichaRds, A. B. (1979). A degrading proteinase test to distinguish benign and virulent ovine isolates of Bacteroides nodosus. Australian Veterinary Journal 55, 25-28.

Egerton, J. R. \& Parsonson, I. M. (1969). Benign foot rot. A specific interdigital dermatitis of sheep associated with infection by less proteolytic strains of Fusiformis nodosus. Australian Veterinary Journal 45, 345-349.

EVERY, D. (1981a). A new method of sample application for horizontal slab polyacrylamide gel electrophoresis. Analytical Biochemistry 115, 7-10.

EvERY, D. $(1981 b)$. Quantitative measurement of protease activity in slab polyacrylamide gel electrophoretograms. Analytical Biochemistry 116, 519 523.

LAING, E. A. \& Egerton, J. R. (1978). The occurrence, prevalence and transmission of Bacteroides nodosus infection in cattle. Research in Veterinary Science 24, 300-304.
Skerman, T. M. (19.75). Determination of some in vitro growth requirements of Bacteroides nodosus. Journal of General Microbiology 87, 107-119.

Skerman, T. M., Erasmuson, S. K. \& Every, D. (1981). Differentiation of Bacteroides nodosus biotypes and colony variants in relation to their virulence and immunoprotective properties in sheep. Infection and Immunity 32, 788-795.

Slater, G. G. (1969). Stable pattern formation and determination of molecular size by pore-limit electrophoresis. Analytical Chemistry 41, 1039-1041.

StewarT, D. J. (1979). The role of elastase in the differentiation of Bacteroides nodosus infections in sheep and cattle. Research in Veterinary Science 27, 99-105.

Thomas, J. H. (1962). The differential diagnosis of foot rot in sheep. Australian Veterinary Journal 38, $159-163$.

Wyckoff, M. Rodbard, D. \& Chrambach, A. (1977). Polyacrylamide gel electrophoresis in sodium dodecyl sulfate-containing buffers using multiphasic buffer systems: properties of the stock, valid Rf-measurement, and optimized procedure. Analytical Biochemistry 78, 459-482. 